\title{
Sharp Bounds of the Hermitian Toeplitz Determinants for Some Classes of Close-to-Convex Functions
}

\author{
Adam Lecko $^{1}$ (D) Barbara Śmiarowska ${ }^{1}$
}

Received: 26 January 2021 / Revised: 17 March 2021 / Accepted: 5 April 2021 / Published online: 16 April 2021

(C) The Author(s) 2021

\section{Abstract}

Sharp upper and lower bounds of the Hermitian Toeplitz determinants of the second and third orders are found for various subclasses of close-to-convex functions.

Keywords Hermitian Toeplitz determinant · Univalent function · Close-to-convex functions · Carathéodory class

Mathematics Subject Classification 30C45 $\cdot 30$ C50

\section{Introduction and Definitions}

Let $\mathbb{D}:=\{z \in \mathbb{C}:|z|<1\}$ and $\overline{\mathbb{D}}:=\{z \in \mathbb{C}:|z| \leq 1\}$, and for $r>0$, let $\mathbb{T}_{r}:=\{z \in \mathbb{C}:|z|=r\}$ and $\mathbb{T}:=\mathbb{T}_{1}$. Denote by $\mathcal{H}$ be the class of all analytic functions $f$ in $\mathbb{D}$ and by $\mathcal{A}$ the subclass of $\mathcal{H}$ with $f$ normalized such that $f(0)=0$ and $f^{\prime}(0)=1$, so that $f(z)$ is of the form

$$
f(z)=\sum_{n=1}^{\infty} a_{n} z^{n}, \quad a_{1}:=1, z \in \mathbb{D} .
$$

Let $\mathcal{S}$ be the subclass of $\mathcal{A}$ consisting of univalent functions.

Communicated by V. Ravichandran.

$\triangle$ Adam Lecko

alecko@matman.uwm.edu.pl

Barbara Śmiarowska

b.smiarowska@matman.uwm.edu.pl

1 Department of Complex Analysis, Faculty of Mathematics and Computer Science, University of Warmia and Mazury in Olsztyn, ul. Słoneczna 54, 10-710 Olsztyn, Poland 
For $q, n \in \mathbb{N}$, consider the matrix $T_{q, n}(f)$ with $f \in \mathcal{A}$ given by (1) defined by

$$
T_{q, n}(f):=\left[\begin{array}{cccc}
a_{n} & a_{n+1} & \ldots & a_{n+q-1} \\
\bar{a}_{n+1} & a_{n} & \ldots & a_{n+q-2} \\
\vdots & \vdots & \vdots & \vdots \\
\bar{a}_{n+q-1} & \bar{a}_{n+q-2} & \ldots & a_{n}
\end{array}\right]
$$

where $\bar{a}_{k}:=\overline{a_{k}}$. In the case when $a_{n}$ is a real number, $T_{q, n}(f)$ is called an Hermitian Toeplitz matrix.

In recent years, a great many papers have been devoted to the estimation of determinants whose entries are coefficients of functions in $\mathcal{A}$ or its subclasses. Hankel matrices, i.e., square matrices which have constant entries along the reverse diagonal and the generalized Zalcman functional $J_{m, n}(f):=a_{m+n-1}-a_{m} a_{n}, m, n \in \mathbb{N}$, are of particular interest (see, e.g., $[5,6,8,13,15,16,18-20,25]$ ). Also of interest are the determinants of symmetric Toeplitz matrices, the study of which was initiated in [1].

In $[9,11,14]$, research was investigated into the study of Hermitian Toeplitz determinants whose entries are the coefficients of functions in subclasses of $\mathcal{A}$.

In this paper, we continue this research by computing the sharp upper and lower bounds of the second- and third-order Hermitian Toeplitz determinants over some subclasses of close-to-convex functions, but first noting that the following general result was proved in [11].

Theorem 1 ([11]) Let $\mathcal{F}$ be a subclass of $\mathcal{A}$ such that $\left\{f \in \mathcal{F}: a_{2}=0\right\} \neq \varnothing$ and $A_{2}(\mathcal{F}):=\max \left\{\left|a_{2}\right|: f \in \mathcal{F}\right\}$ exists. Then

$$
1-A_{2}^{2}(\mathcal{F}) \leq \operatorname{det} T_{2,1}(f) \leq 1
$$

Both inequalities are sharp.

We next define the classes of close-to-convex functions considered in this paper. First denote by $\mathcal{S}^{*}$ the subclass of $\mathcal{S}$ consisting of the starlike functions, i.e., $f \in \mathcal{S}^{*}$ if and only if $f \in \mathcal{A}$ and

$$
\operatorname{Re} \frac{z f^{\prime}(z)}{f(z)}>0, \quad z \in \mathbb{D} .
$$

A function $f \in \mathcal{A}$ is called close-to-convex if there exist $g \in \mathcal{S}^{*}$ and $\delta \in \mathbb{R}$ such that

$$
\operatorname{Re} \frac{\mathrm{e}^{\mathrm{i} \delta} z f^{\prime}(z)}{g(z)}>0, \quad z \in \mathbb{D} .
$$

The class $\mathcal{C}$ of all close-to-convex functions (which is necessarily a subclass $\mathcal{S}$ ), was introduced by Kaplan [12] (see also [10, Vol. II, p. 3]), where the following geometrical interpretation was given: $f \in \mathcal{A}$ is close-to-convex if and only if there are no sections of the curve $f\left(\mathbb{T}_{r}\right)$, for every $r \in(0,1)$, in which tangent vector turns backward through an angle not less than $\pi$ (cf. [10, Vol. II, p. 4]). Lewandowski [22,23] proved that 
the class of close-to-convex functions is identical with the class of linearly accessible functions introduced by Biernacki [3].

Given $g \in \mathcal{S}^{*}$ and $\delta \in \mathbb{R}$, let $\mathcal{C}_{\delta}(g)$ be the subclass of $\mathcal{C}$ of all $f$ satisfying (2). The four classes $\mathcal{C}_{0}\left(g_{i}\right), i=1, \ldots, 4$, where

$$
g_{1}(z):=\frac{z}{1-z^{2}}, \quad g_{2}(z):=\frac{z}{(1-z)^{2}}, \quad g_{3}(z):=\frac{z}{1+z+z^{2}}
$$

and

$$
g_{4}(z):=\frac{z}{1-z}, \quad z \in \mathbb{D}
$$

are particularly interesting and have been studied by various authors (e.g., [2,7,17]). In [14], the sharp bounds of the second- and third-order Hermitian Toeplitz determinants were found for the classes $\mathcal{C}_{0}\left(g_{1}\right)$ and $\mathcal{C}_{0}\left(g_{2}\right)$. In this paper, we will do the same for the other two classes, i.e., for $\mathcal{C}_{0}\left(g_{3}\right)=: \mathcal{F}_{1}$ and $\mathcal{C}_{0}\left(g_{4}\right)=: \mathcal{F}_{2}$ which $f$ in view of (2) satisfy the conditions

$$
\operatorname{Re}\left\{\left(1+z+z^{2}\right) f^{\prime}(z)\right\}>0, \quad z \in \mathbb{D}
$$

and

$$
\operatorname{Re}\left\{(1-z) f^{\prime}(z)\right\}>0, \quad z \in \mathbb{D},
$$

respectively. We note here that in [21] the $\operatorname{classes} \mathcal{C}\left(\delta, \xi_{1}, \xi_{2}\right)$, where $\delta \in(-\pi / 2, \pi / 2)$, $\xi_{1}, \xi_{2} \in \overline{\mathbb{D}}$, of univalent functions were introduced by generalizing Robertson's condition for convexity in the direction of the imaginary axis [26]. In particular, the class $\mathcal{C}(0,(-1-\sqrt{3} i) / 2,(-1+\sqrt{3} i) / 2)$ is identical to the class $\mathcal{F}_{1}$ and the class $\mathcal{C}(0,0,1)$ is identical to the class $\mathcal{F}_{2}$. A geometrical property of functions in classes $\mathcal{C}\left(\delta, \xi_{1}, \xi_{2}\right)$ relating to the hyperbolic or parabolic family of arcs related to $\xi_{1}$ and $\xi_{2}$ was presented in [21].

Let $\mathcal{P}$ be the class of all $p \in \mathcal{H}$ of the form

$$
p(z)=1+\sum_{n=1}^{\infty} c_{n} z^{n}, \quad z \in \mathbb{D},
$$

having a positive real part in $\mathbb{D}$.

\section{Lemmas}

In the proof of our main result, we will use the following lemma, see ([4], [24, p. 166]).

Lemma 1 If $p \in \mathcal{P}$ is of the form (5), then

$$
\left|c_{n}\right| \leq 2, \quad n \in \mathbb{N} \text {. }
$$


Moreover,

$$
c_{1}=2 \zeta_{1}
$$

and

$$
c_{2}=2 \zeta_{1}^{2}+2\left(1-\left|\zeta_{1}\right|^{2}\right) \zeta_{2}
$$

for some $\zeta_{i} \in \overline{\mathbb{D}}, i \in\{1,2\}$.

For $\zeta_{1} \in \mathbb{T}$, there is a unique function $p \in \mathcal{P}$ with $c_{1}$ as in (7), namely

$$
p(z)=\frac{1+\zeta_{1} z}{1-\zeta_{1} z}, \quad z \in \mathbb{D}
$$

For $\zeta_{1} \in \mathbb{D}$ and $\zeta_{2} \in \mathbb{T}$, there is a unique function $p \in \mathcal{P}$ with $c_{1}$ and $c_{2}$ as in (7) and (8), namely

$$
p(z)=\frac{1+\left(\bar{\zeta}_{1} \zeta_{2}+\zeta_{1}\right) z+\zeta_{2} z^{2}}{1+\left(\bar{\zeta}_{1} \zeta_{2}-\zeta_{1}\right) z-\zeta_{2} z^{2}}, \quad z \in \mathbb{D}
$$

\section{The Class $\mathcal{F}_{1}$}

Let $f \in \mathcal{F}_{1}$ be the form (1). Then by (3) there exists $p \in \mathcal{P}$ of the form (5) such that

$$
\left(1+z+z^{2}\right) f^{\prime}(z)=p(z), \quad z \in \mathbb{D}
$$

Substituting (1) and (5) into (10) and equating the coefficients, we obtain

$$
a_{2}=\frac{1}{2}\left(-1+c_{1}\right), \quad a_{3}=\frac{1}{3}\left(-c_{1}+c_{2}\right) .
$$

Hence, using (6) it follows that $A_{2}\left(\mathcal{F}_{1}\right)=3 / 2$ with $f_{1} \in \mathcal{F}_{1}$ satisfying

$$
\left(1+z+z^{2}\right) f_{1}^{\prime}(z)=\frac{1-z}{1+z}, \quad z \in \mathbb{D},
$$

i.e.,

$$
f_{1}(z)=\int_{0}^{z} \frac{1-\xi}{1+2 \xi+2 \xi^{2}+\xi^{3}} d \xi=z-\frac{3}{2} z^{2}+\cdots, \quad z \in \mathbb{D} .
$$

Also $f_{2} \in \mathcal{F}_{1}$ such that

$$
\left(1+z+z^{2}\right) f_{2}^{\prime}(z)=\frac{1}{1-z}, \quad z \in \mathbb{D},
$$

i.e.,

$$
f_{2}(z)=\int_{0}^{z} \frac{d \xi}{1-\xi^{3}}=z+\frac{1}{4} z^{4}+\cdots, \quad z \in \mathbb{D},
$$

serves as the extreme function, since $a_{2}=0$. Thus, Theorem 1 gives 
Theorem 2 If $f \in \mathcal{F}_{1}$, then

$$
-\frac{5}{4} \leq \operatorname{det} T_{2,1}(f) \leq 1
$$

Both inequalities are sharp.

We now find the upper and lower bounds of $\operatorname{det} T_{3,1}(f)$ in the class $\mathcal{F}_{1}$, first noting that

$$
\operatorname{det} T_{3,1}(f)=\left|\begin{array}{ccc}
1 & a_{2} & a_{3} \\
\bar{a}_{2} & 1 & a_{2} \\
\bar{a}_{3} & \bar{a}_{2} & 1
\end{array}\right|=2 \operatorname{Re}\left(a_{2}^{2} \bar{a}_{3}\right)-2\left|a_{2}\right|^{2}-\left|a_{3}\right|^{2}+1
$$

Theorem 3 If $f \in \mathcal{F}_{1}$, then

$$
-1 \leq \operatorname{det} T_{3,1}(f) \leq 1
$$

Both inequalities are sharp.

Proof We first find the upper bound.

By (11) and (6), we see that $\left|a_{2}\right| \leq 3 / 2$ and $\left|a_{3}\right| \leq 4 / 3$. Since $\operatorname{Re}\left(a_{2}^{2} \bar{a}_{3}\right) \leq\left|a_{2}\right|^{2}\left|a_{3}\right|$, it follows from (13) that

$$
\operatorname{det} T_{3,1}(f) \leq F\left(\left|a_{2}\right|,\left|a_{3}\right|\right)
$$

where

$$
F(x, y):=2 x^{2} y-2 x^{2}-y^{2}+1, \quad(x, y) \in[0,3 / 2] \times[0,4 / 3] .
$$

Observe now that the point $(1,1)$ is the unique solution in $(0,3 / 2) \times(0,4 / 3)$ of the system of equations

$$
\begin{aligned}
& \frac{\partial F}{\partial x}=4 x(y-1)=0, \\
& \frac{\partial F}{\partial y}=2\left(x^{2}-y\right)=0 .
\end{aligned}
$$

However,

$$
\frac{\partial^{2} F}{\partial x^{2}}(1,1) \frac{\partial^{2} F}{\partial y^{2}}(1,1)-\left(\frac{\partial F}{\partial x \partial y}(1,1)\right)^{2}=-16<0
$$

so $(1,1)$ is a saddle point of $F$.

We now consider $F$ on the boundary of $[0,3 / 2] \times[0,4 / 3]$.

(1) On the side $x=0$,

$$
F(0, y)=1-y^{2} \leq 1, \quad 0 \leq y \leq \frac{4}{3} .
$$


(2) On the side $x=3 / 2$,

$$
F\left(\frac{3}{2}, y\right)=-\frac{7}{2}+\frac{9}{2} y-y^{2} \leq F\left(\frac{3}{2}, \frac{4}{3}\right)=\frac{13}{18}, \quad 0 \leq y \leq \frac{4}{3} .
$$

(3) On the side $y=0$,

$$
F(x, 0)=1-2 x^{2} \leq 1, \quad 0 \leq x \leq \frac{3}{2} .
$$

(4) On the side $y=4 / 3$

$$
F\left(x, \frac{4}{3}\right)=-\frac{7}{9}+\frac{2}{3} x^{2} \leq \frac{13}{18}, \quad 0 \leq x \leq \frac{3}{2}
$$

Therefore, the inequality $F(x, y) \leq 1$ holds for all $(x, y) \in[0,3 / 2] \times[0,4 / 3]$, which in view of (15) gives the upper bound.

For the lower bound, we substitute (7) and (8) into (11) to obtain

$$
a_{2}=\frac{1}{2}\left(-1+2 \zeta_{1}\right), \quad a_{3}=\frac{1}{3}\left[-2 \zeta_{1}+2 \zeta_{1}^{2}+2\left(1-\left|\zeta_{1}\right|^{2}\right) \zeta_{2}\right]
$$

with $\zeta_{i} \in \overline{\mathbb{D}}, i=1,2$. Therefore, from (13) we have

$$
\operatorname{det} T_{3,1}(f)=\frac{1}{18}\left(\Psi_{1}+\Psi_{2}\right)
$$

where

$$
\Psi_{1}:=9-20\left|\zeta_{1}\right|^{2}+16\left|\zeta_{1}\right|^{4}-8\left(1-\left|\zeta_{1}\right|^{2}\right)^{2}\left|\zeta_{2}\right|^{2}
$$

and

$$
\begin{aligned}
\Psi_{2}:= & 30 \operatorname{Re} \zeta_{1}+6 \operatorname{Re}\left(\zeta_{1}^{2}\right)-32\left|\zeta_{1}\right|^{2} \operatorname{Re} \zeta_{1}-8\left(1-\left|\zeta_{1}\right|^{2}\right) \operatorname{Re}\left(\zeta_{1} \bar{\zeta}_{2}\right) \\
& +6\left(1-\left|\zeta_{1}\right|^{2}\right) \operatorname{Re} \zeta_{2}+8\left(1-\left|\zeta_{1}\right|^{2}\right) \operatorname{Re}\left(\zeta_{1}^{2} \bar{\zeta}_{2}\right)
\end{aligned}
$$

We now consider various cases.

A. Suppose that $\zeta_{1} \zeta_{2} \neq 0$. Then $\zeta_{1}=r \mathrm{e}^{\mathrm{i} \theta}$ and $\zeta_{2}=s \mathrm{e}^{\mathrm{i} \psi}$ with $r, s \in(0,1]$ and $\theta, \psi \in[0,2 \pi)$. Then

$$
\Psi_{2}=\Psi_{3}+\Psi_{4},
$$

where

$$
\begin{aligned}
\Psi_{3} & :=30 r \cos \theta+6 r^{2} \cos 2 \theta-32 r^{3} \cos \theta \\
& =-6 r^{2}+30 r \cos \theta-32 r^{3} \cos \theta+12 r^{2} \cos ^{2} \theta
\end{aligned}
$$


and

$$
\begin{aligned}
\Psi_{4} & :=-8 r s\left(1-r^{2}\right) \cos (\theta-\psi)+6 s\left(1-r^{2}\right) \cos \psi+8 r^{2} s\left(1-r^{2}\right) \cos (2 \theta-\psi) \\
& =2 s\left(1-r^{2}\right) \sqrt{\kappa_{1}^{2}+\kappa_{2}^{2}} \sin (\psi+\alpha),
\end{aligned}
$$

where $\alpha \in \mathbb{R}$ satisfies

$$
\cos \alpha=\frac{\kappa_{1}}{\sqrt{\kappa_{1}^{2}+\kappa_{2}^{2}}}, \quad \sin \alpha=\frac{\kappa_{2}}{\sqrt{\kappa_{1}^{2}+\kappa_{2}^{2}}}
$$

with

$$
\kappa_{1}:=-4 r \sin \theta+4 r^{2} \sin 2 \theta, \quad \kappa_{2}:=3-4 r \cos \theta+4 r^{2} \cos 2 \theta .
$$

Since $\sin (\psi+\alpha) \geq-1$ and $s \leq 1$, we have

$$
\begin{aligned}
\Psi_{4} & \geq-2\left(1-r^{2}\right) \sqrt{\kappa_{1}^{2}+\kappa_{2}^{2}} \\
& =-2\left(1-r^{2}\right) \sqrt{9-8 r^{2}+16 r^{4}-24 r \cos \theta-32 r^{3} \cos \theta+48 r^{2} \cos ^{2} \theta}
\end{aligned}
$$

Therefore, from (18)-(20), we obtain

$$
\begin{aligned}
\Psi_{2}= & \Psi_{3}+\Psi_{4} \\
\geq & 30 r \cos \theta+6 r^{2} \cos 2 \theta-32 r^{3} \cos \theta \\
& -2\left(1-r^{2}\right) \sqrt{9-8 r^{2}+16 r^{4}-24 r \cos \theta-32 r^{3} \cos \theta+48 r^{2} \cos ^{2} \theta}
\end{aligned}
$$

Noting that $\left|\zeta_{2}\right| \leq 1$ from (17) we have

$$
\Psi_{1} \geq 1-4 r^{2}+8 r^{4}
$$

Thus, from (16), (23) and (24) it follows that

$$
18 \operatorname{det} T_{3,1}(f) \geq G(r, \cos \theta), \quad r \in(0,1], \theta \in[0,2 \pi),
$$

where

$$
G(t, x):=g_{1}(t, x)-2\left(1-t^{2}\right) \sqrt{g_{2}(t, x)}
$$

with

$$
g_{1}(t, x):=1-10 t^{2}+8 t^{4}+30 t x-32 t^{3} x+12 t^{2} x^{2}
$$

and

$$
g_{2}(t, x):=9-8 t^{2}+16 t^{4}-24 t x-32 t^{3} x+48 t^{2} x^{2}
$$


for $t \in[0,1]$ and $x \in[-1,1]$.

Let $\Omega:=[0,1] \times[-1,1]$. Now we will show that

$$
\min \{G(t, x):(t, x) \in \Omega\}=-18 .
$$

A1. We next deal with the critical points of $G$ in the interior of $\Omega$, i.e., in $(0,1) \times$ $(-1,1)$. Note that $g_{2}(t, x) \geq 0$. Moreover, $g_{2}(t, x)=0$ holds only for $t=\sqrt{3} / 2$ and $x=\sqrt{3} / 3$.

A1.1. When $t=\sqrt{3} / 2$ and $x=\sqrt{3} / 3$, from (17), (19)and (20) we have

$$
\Psi_{1}=3-\frac{1}{2}\left|\zeta_{2}\right|^{2}, \quad \Psi_{3}=\frac{3}{2}, \quad \Psi_{4}=0
$$

which gives

$$
\operatorname{det} T_{3,1}(f)=-\frac{1}{36}\left(-9+\left|\zeta_{2}\right|^{2}\right) \geq \frac{2}{9} .
$$

A1.2. We now consider case $g_{2}(t, x)>0$. Differentiating $G$ with respect to $x$ yields

$$
0=\frac{\partial G}{\partial x}(t, x)=\frac{\partial g_{1}}{\partial x}(t, x)-\left(1-t^{2}\right)\left(g_{2}(t, x)\right)^{-1 / 2} \frac{\partial g_{2}}{\partial x}(t, x) .
$$

A1.2.a. Assume first that $\partial g_{1} / \partial x=0$. Then from (27), it follows that $\partial g_{2} / \partial x=0$, which is possible only for $t=\sqrt{6} / 2>1$ and $x=\sqrt{6} / 4$.

A1.2.b. Assume next that $\partial g_{1} / \partial x \neq 0$. Then we can write (27) as

$$
g_{2}(t, x)^{1 / 2}=\frac{\left(1-t^{2}\right) \frac{\partial g_{2}}{\partial x}(t, x)}{\frac{\partial g_{1}}{\partial x}(t, x)}=\frac{4\left(1-t^{2}\right)\left(-3-4 t^{2}+12 t x\right)}{15-16 t^{2}+12 t x}
$$

or equivalently, by substituting (26), as

$$
\begin{aligned}
\Phi=\Phi(t, x):= & 3840 t^{8}-12800 t^{7} x+24576 t^{6} x^{2}-23040 t^{5} x^{3}+6912 t^{4} x^{4} \\
& -9600 t^{6}+16128 t^{5} x-21888 t^{4} x^{2}+13824 t^{3} x^{3}+10112 t^{4} \\
& -2784 t^{3} x+1152 t^{2} x^{2}-6216 t^{2}-1008 t x+1881=0 .
\end{aligned}
$$

Now note that by (28) the inequality

$$
0<\frac{\left(g_{2}(t, x)\right)^{1 / 2}}{4\left(1-t^{2}\right)}=\frac{-3-4 t^{2}+12 t x}{15-16 t^{2}+12 t x}
$$

holds for

$$
0<t<\sqrt{\frac{3}{2}} \text { and } \frac{4 t^{2}+3}{12 t}<x<\frac{16 t^{2}-15}{12 t}
$$


Differentiating $G$ with respect to $t$, we have

$$
\frac{\partial G}{\partial t}(t, x)=\frac{\partial g_{1}}{\partial t}(t, x)+4 t\left(g_{2}(t, x)\right)^{1 / 2}-\left(1-t^{2}\right)\left(g_{2}(t, x)\right)^{-1 / 2} \frac{\partial g_{2}}{\partial t}(t, x) .
$$

By (28) and (31), we get

$$
\frac{\partial G}{\partial t}(t, x)=-\frac{72 t}{\left(12 x t-4 t^{2}-3\right)\left(12 x t-16 t^{2}+15\right)} H(t, x),
$$

where for $(t, x) \in(0,1) \times(-1,1)$,

$$
H(t, x):=\left(-3+4 t^{2}-4 t x\right)\left(9-18 t^{2}+8 t^{4}+6 t x+8 t^{3} x-24 t^{2} x^{2}\right) .
$$

Therefore, each critical point of $G$ satisfies

$$
-3+4 t^{2}-4 t x=0
$$

or

$$
9-18 t^{2}+8 t^{4}+6 t x+8 t^{3} x-24 t^{2} x^{2}=0 .
$$

I. Assume that (32) holds, then $x=x(t)=\left(-3+4 t^{2}\right) /(4 t)$. Thus, by (29) we see that

$$
\Phi\left(t, \frac{-3+4 t^{2}}{4 t}\right)=-8(2 t+1)(2 t-1)\left(4 t^{2}-5\right)\left(2 t^{2}-3\right)^{2}=0
$$

occurs only when $t=1 / 2$. Thus, $x=x(1 / 2)=-1$. However, it can be seen that the right side of (30) equals to -2 for $t=1 / 2$ and $x=-1$, which means that then the inequality (30) is not true. Therefore, $G$ does not have critical point in the interior of $\Omega$ in the case of (32).

II. Suppose now that (33) is satisfied. Since Eq. (33) is a quadratic in $x$ with $\Delta:=$ $225-408 t^{2}+208 t^{4}>0, t \in(0,1)$, it has two roots, namely

$$
x_{i}=x_{i}(t)=\frac{3+4 t^{2}+(-1)^{i+1} \sqrt{225-408 t^{2}+208 t^{4}}}{24 t}, \quad i=1,2 .
$$

a. Let $x=x_{1}$. Then $\Phi\left(t, x_{1}(t)\right)=0$ is equivalent to the equation

$$
\begin{aligned}
& \left(-561+2204 t^{2}-2736 t^{4}+1088 t^{6}\right) \sqrt{225-408 t^{2}+208 t^{4}} \\
& =11169-48552 t^{2}+80288 t^{4}-59520 t^{6}+16640 t^{8}
\end{aligned}
$$

Squaring both sides of (35) leads to

$$
2304 \gamma_{1}(t) \gamma_{2}^{2}(t)=0
$$


where for $t \in(0,1)$,

$$
\gamma_{1}(t):=289-408 t^{2}+208 t^{4}
$$

and

$$
\gamma_{2}(t):=9-27 t^{2}+26 t^{4}-8 t^{6}=-(1-t)(1+t)\left(3-4 t^{2}\right)\left(3-2 t^{2}\right) .
$$

We see that there is a unique $\operatorname{root} t=\sqrt{3} / 2$ of the Eq. (36), which also satisfies (35). Then by (34), $x_{1}(\sqrt{3} / 2)=\sqrt{3} / 3$. However, this case was discussed in A1.1.

b. Let $x=x_{2}$. Then $\Phi\left(t, x_{2}(t)\right)=0$ is equivalent to the equation

$$
\begin{aligned}
& -\left(-561+2204 t^{2}-2736 t^{4}+1088 t^{6}\right) \sqrt{225-408 t^{2}+208 t^{4}} \\
& =11169-48552 t^{2}+80288 t^{4}-59520 t^{6}+16640 t^{8}
\end{aligned}
$$

Squaring both sides of (37) yields again the Eq. (36) having a unique root $t=\sqrt{3} / 2$, which does not satisfy (37)

A2. It therefore remains to consider $G$ on the boundary of $\Omega$.

(1) On the side $t=0$,

$$
G(0, x) \equiv-5, \quad x \in[-1,1] .
$$

(2) On the side $t=1$,

$$
G(1, x)=-1-2 x+12 x^{2} \geq G\left(1, \frac{1}{12}\right)=-\frac{13}{12}, \quad x \in[-1,1] .
$$

(3) On the side $x=-1$,

$$
G(t,-1)=-5-38 t+40 t^{3}+16 t^{4}=: \varrho_{1}(t), \quad t \in[0,1]
$$

Since $\varrho_{1}^{\prime}(t)=0$ occurs only when $t=1 / 2$ and $\varrho_{1}^{\prime \prime}(1 / 2)=168>0$, we have

$$
\varrho_{1}(t) \geq \varrho_{1}(1 / 2)=-18, \quad t \in[0,1]
$$

(4) On the side $x=1$,

$$
G(t, 1)=-5+38 t-40 t^{3}+16 t^{4}=: \varrho_{2}(t), \quad t \in[0,1] .
$$

Since $\varrho_{2}^{\prime}(t)=0$ occurs only when $t=(19-\sqrt{57}) / 16 \in[0,1]$ and $\varrho_{2}^{\prime \prime}((19-$ $\sqrt{57}) / 16)=(57-27 \sqrt{57}) / 2<0$, we have

$$
\varrho_{2}(t) \geq \varrho_{2}(0)=-5, \quad t \in[0,1]
$$


B. Suppose that $\zeta_{1}=0$. Then

$$
\Psi_{1}=9-8\left|\zeta_{2}\right|^{2}, \quad \Psi_{2}=6 \operatorname{Re} \zeta_{2}
$$

and therefore,

$$
\operatorname{det} T_{3,1}(f)=\frac{1}{18}\left(9+6 \operatorname{Re} \zeta_{2}-8\left|\zeta_{2}\right|^{2}\right) \geq-\frac{5}{18}
$$

C. Suppose that $\zeta_{2}=0$ and $\zeta_{1}=r \mathrm{e}^{\mathrm{i} \theta} \neq 0$, where $r \in(0,1]$ and $\theta \in[0,2 \pi)$. Then

$$
\Psi_{1}=9-20\left|\zeta_{1}\right|^{2}+16\left|\zeta_{1}\right|^{4}=9-20 r^{2}+16 r^{4}
$$

and

$\Psi_{2}=30 \operatorname{Re} \zeta_{1}+6 \operatorname{Re}\left(\zeta_{1}^{2}\right)-32\left|\zeta_{1}\right|^{2} \operatorname{Re} \zeta_{1}=30 r \cos \theta+6 r^{2} \cos 2 \theta-32 r^{3} \cos \theta$

Thus,

$$
18 \operatorname{det} T_{3,1}(f)=G(r, \cos \theta), \quad r \in(0,1], \theta \in[0,2 \pi),
$$

where

$$
G(t, x):=9-26 t^{2}+16 t^{4}+30 t x-32 t^{3} x+12 t^{2} x^{2}
$$

for $t \in(0,1]$ and $x \in[-1,1]$. Set

$$
x_{w}:=-\frac{15-16 t^{2}}{12 t}, \quad t \in(0,1] .
$$

Note that $-1<x_{w}$ occurs for $t>(\sqrt{69}-3) / 8=0.663328 \cdots$, and $x_{w}<1$ holds for $t \in(0,1)$. Hence, for $t \in((\sqrt{69}-3) / 8,1)$ we have

$$
G(t, x) \geq G\left(t, x_{w}\right)=-\frac{39}{4}+14 t^{2}-\frac{16}{3} t^{4}=: \phi_{1}(t) .
$$

Since $\phi_{1}$ is increasing in $((\sqrt{69}-3) / 8,1)$, so

$$
\phi_{1}(t) \geq-\frac{1}{32}(123+3 \sqrt{69})=-4.622495 \cdots
$$

for $t \in((\sqrt{69}-3) / 8,1)$. Further, $x_{w}<-1$ occurs for $t \in(0,(\sqrt{69}-3) / 8)$. Hence,

$$
G(t, x) \geq G(t,-1)=9-30 t-14 t^{2}+32 t^{3}+16 t^{4}=: \phi_{2}(t) .
$$


Since $\phi_{2}^{\prime}(t)=0$ have a unique root $t_{0}=(-2+\sqrt{19}) / 4=0.589724 \cdots$ and $\phi_{2}^{\prime \prime}\left(t_{0}\right)=$ $152>0$, then

$\phi_{2}(t) \geq \phi_{2}\left(\frac{1}{4}(-2+\sqrt{19})\right)=-\frac{81}{16}=-5.062500 \cdots, \quad t \in\left(0, \frac{1}{8}(\sqrt{69}-3)\right)$.

Summarizing, form Parts A-C it follows the lower bound in (14).

We discuss now sharpness of (14). The function $f_{2}$ defined by (12), for which $a_{2}=a_{3}=0$, is extremal for the upper bound in (14). It is observed from (16), (23), (24) and (25) that equality for the lower bound in (14) holds when the following conditions are satisfied:

$$
r=\frac{1}{2}, \quad \cos \theta=-1, \quad s=1, \quad \sin (\psi+\alpha)=-1,
$$

where $\alpha$ is determined by the condition (21) with $\kappa_{1}$ and $\kappa_{2}$ given in (22). Thus, $\theta=\pi$, $\alpha=\pi / 2$ and $\psi=\pi$. Consequently, $\zeta_{1}=-1 / 2$ and $\zeta_{2}=-1$, which in view of (9) holds for the function

$$
p(z)=\frac{1-z^{2}}{1+z+z^{2}}, \quad z \in \mathbb{D}
$$

in the class $\mathcal{P}$. Therefore, the extremal function $f$ in the class $\mathcal{F}_{1}$ for the lower bound in (14) satisfies (10) with $p$ given as above, having the coefficients $a_{2}=-1$ and $a_{3}=0$.

\section{The Class $\mathcal{F}_{2}$}

Let $f \in \mathcal{F}_{2}$ be the form (1). Then by (4) there exists $p \in \mathcal{P}$ of the form (5) such that

$$
(1-z) f^{\prime}(z)=p(z), \quad z \in \mathbb{D} \text {. }
$$

Putting the series (1) and (5) into (40) by equating the coefficients, we get

$$
a_{2}=\frac{1}{2}\left(1+c_{1}\right), \quad a_{3}=\frac{1}{3}\left(1+c_{1}+c_{2}\right) .
$$

Hence and by (6), it follows that $A_{2}\left(\mathcal{F}_{2}\right)=3 / 2$ with the extremal function $f_{1} \in \mathcal{F}_{2}$ such that

$$
(1-z) f_{1}^{\prime}(z)=\frac{1+z}{1-z}, \quad z \in \mathbb{D} .
$$

Observe also that $a_{2}=0$ for the function $f_{2} \in \mathcal{F}_{2}$ such that

$$
(1-z) f_{2}^{\prime}(z)=\frac{1}{1+z}, \quad z \in \mathbb{D}
$$

Therefore, by Theorem 1 we have 
Theorem 4 If $f \in \mathcal{F}_{2}$, then

$$
-\frac{5}{4} \leq \operatorname{det} T_{2,1}(f) \leq 1
$$

Both inequalities are sharp.

Now we estimate det $T_{3,1}(f)$ for functions in the class $\mathcal{F}_{2}$.

Theorem 5 If $f \in \mathcal{F}_{2}$, then

$$
\operatorname{det} T_{3,1}(f) \leq \frac{11}{9}
$$

The inequality is sharp.

Proof By (41) and (6), we see that $\left|a_{2}\right| \leq 3 / 2$ and $\left|a_{3}\right| \leq 5 / 3$. As in the proof of Theorem 3, the inequality (15) holds with the function

$$
F(x, y):=2 x^{2} y-2 x^{2}-y^{2}+1, \quad(x, y) \in[0,3 / 2] \times[0,5 / 3]
$$

Repeating argumentation in the proof of Theorem 3, we see that the function $F$ does not have any relative maxima in $(0,3 / 2) \times(0,5 / 3)$.

We consider $F$ on the boundary of $[0,3 / 2] \times[0,5 / 3]$.

(1) On the side $x=0$,

$$
F(0, y)=1-y^{2} \leq 1, \quad 0 \leq y \leq \frac{5}{3}
$$

(2) On the side $x=3 / 2$,

$$
F\left(\frac{3}{2}, y\right)=-\frac{7}{2}+\frac{9}{2} y-y^{2} \leq F\left(\frac{3}{2}, \frac{5}{3}\right)=\frac{11}{9}, \quad 0 \leq y \leq \frac{5}{3} .
$$

(3) On the side $y=0$,

$$
F(x, 0)=1-2 x^{2} \leq 1, \quad 0 \leq x \leq \frac{3}{2} .
$$

(4) On the side $y=5 / 3$,

$$
F\left(x, \frac{5}{3}\right)=-\frac{16}{9}+\frac{4}{3} x^{2} \leq F\left(\frac{3}{2}, \frac{5}{3}\right)=\frac{11}{9}, \quad 0 \leq x \leq \frac{3}{2}
$$

Therefore, the inequality $F(x, y) \leq 11 / 9$ holds for all $(x, y) \in[0,3 / 2] \times[0,5 / 3]$ which in view of (15) shows (43).

For the function $f_{1}$ given by (42), $a_{2}=3 / 2$ and $a_{3}=5 / 3$ which makes equality in (43). 
Theorem 6 If $f \in \mathcal{F}_{2}$, then

$$
\operatorname{det} T_{3,1}(f) \geq \frac{1}{44}(32-31 \sqrt{3})=-0.493035 \cdots
$$

The inequality is sharp.

Proof Substituting (7) and (8) into (41) yields

$$
a_{2}=\frac{1}{2}\left(2 \zeta_{1}+1\right), \quad a_{3}=\frac{1}{3}\left[1+2 \zeta_{1}+2 \zeta_{1}^{2}+2\left(1-\left|\zeta_{1}\right|^{2}\right) \zeta_{2}\right],
$$

for some $\zeta_{i} \in \overline{\mathbb{D}}(i=1,2)$. Therefore, from (13) we get

$$
\operatorname{det} T_{3,1}(f)=\frac{1}{18}\left(\Psi_{1}+\Psi_{2}\right)
$$

where

$$
\Psi_{1}:=10-20\left|\zeta_{1}\right|^{2}+16\left|\zeta_{1}\right|^{4}-8\left(1-\left|\zeta_{1}\right|^{2}\right)^{2}\left|\zeta_{2}\right|^{2}
$$

and

$$
\begin{aligned}
\Psi_{2}:= & -26 \operatorname{Re} \zeta_{1}+10 \operatorname{Re}\left(\zeta_{1}^{2}\right)+32\left|\zeta_{1}\right|^{2} \operatorname{Re} \zeta_{1}+8\left(1-\left|\zeta_{1}\right|^{2}\right) \operatorname{Re}\left(\zeta_{1} \bar{\zeta}_{2}\right) \\
& -2\left(1-\left|\zeta_{1}\right|^{2}\right) \operatorname{Re} \zeta_{2}+8\left(1-\left|\zeta_{1}\right|^{2}\right) \operatorname{Re}\left(\zeta_{1}^{2} \bar{\zeta}_{2}\right) .
\end{aligned}
$$

A. Suppose that $\zeta_{1} \zeta_{2} \neq 0$. Thus, $\zeta_{1}=r \mathrm{e}^{\mathrm{i} \theta}$ and $\zeta_{2}=s \mathrm{e}^{\mathrm{i} \psi}$ with $r, s \in(0,1]$ and $\theta, \psi \in[0,2 \pi)$. Then

$$
\Psi_{2}=2\left(\Psi_{3}+\Psi_{4}\right)
$$

where

$$
\begin{aligned}
\Psi_{3} & :=-13 r \cos \theta+5 r^{2} \cos 2 \theta+16 r^{3} \cos \theta \\
& =-5 r^{2}-13 r \cos \theta+16 r^{3} \cos \theta+10 r^{2} \cos ^{2} \theta
\end{aligned}
$$

and

$$
\begin{aligned}
\Psi_{4} & :=4 r s\left(1-r^{2}\right) \cos (\theta-\psi)-s\left(1-r^{2}\right) \cos \psi+4 r^{2} s\left(1-r^{2}\right) \cos (2 \theta-\psi) \\
& =s\left(1-r^{2}\right) \sqrt{\kappa_{1}^{2}+\kappa_{2}^{2}} \sin (\psi+\alpha),
\end{aligned}
$$

where $\alpha$ is the quantity satisfying (21) with

$$
\kappa_{1}:=4 r \sin \theta+4 r^{2} \sin 2 \theta, \quad \kappa_{2}:=-1+4 r \cos \theta+4 r^{2} \cos 2 \theta .
$$

Since $\sin (\psi+\alpha) \geq-1$ and $s \leq 1$, we have

$$
\begin{aligned}
\Psi_{4} & \geq-\left(1-r^{2}\right) \sqrt{\kappa_{1}^{2}+\kappa_{2}^{2}} \\
& =-\left(1-r^{2}\right) \sqrt{1+24 r^{2}+16 r^{4}-8 r \cos \theta+32 r^{3} \cos \theta-16 r^{2} \cos ^{2} \theta} .
\end{aligned}
$$


Therefore, from (47), (48) and (49), we get

$$
\begin{aligned}
\frac{1}{2} \Psi_{2}= & \Psi_{3}+\Psi_{4} \\
\geq & -13 r \cos \theta+5 r^{2} \cos 2 \theta+16 r^{3} \cos \theta \\
& -\left(1-r^{2}\right) \sqrt{1+24 r^{2}+16 r^{4}-8 r \cos \theta+32 r^{3} \cos \theta-16 r^{2} \cos ^{2} \theta}
\end{aligned}
$$

Taking into account that $\left|\zeta_{2}\right| \leq 1$ from (46) we have

$$
\Psi_{1} \geq 2\left(1-2 r^{2}+4 r^{4}\right)
$$

Thus, from (45), (51) and (52) it follows that

$$
9 \operatorname{det} T_{3,1}(f) \geq G(r, \cos \theta), \quad r \in(0,1], \theta \in[0,2 \pi),
$$

where

$$
G(t, x):=g_{1}(t, x)-\left(1-t^{2}\right) \sqrt{g_{2}(t, x)}
$$

with

$$
g_{1}(t, x):=1-7 t^{2}+4 t^{4}-13 t x+16 t^{3} x+10 t^{2} x^{2}
$$

and

$$
g_{2}(t, x):=1+24 t^{2}+16 t^{4}-8 t x+32 t^{3} x-16 t^{2} x^{2}
$$

for $t \in[0,1]$ and $x \in[-1,1]$.

Let $\Omega:=[0,1] \times[-1,1]$ and

$$
\Theta:=\frac{9}{44}(32-31 \sqrt{3})=-4.4373 \cdots
$$

Now we will show that

$$
\min \{G(t, x):(t, x) \in \Omega\}=\Theta .
$$

A1. For this, we first we find the critical points of $G$ in the interior of $\Omega$, i.e., in $(0,1) \times(-1,1)$. Since $g_{2}(t, x)=0$ holds only for $t=(\sqrt{2}-1) / 2$ and $x=1$, it follows that $g_{2}(t, x)>0$ for $(t, x) \in(0,1) \times(-1,1)$.

Differentiating $G$ with respect to $x$ yields

$$
0=\frac{\partial G}{\partial x}(t, x)=\frac{\partial g_{1}}{\partial x}(t, x)-\frac{1}{2}\left(1-t^{2}\right)\left(g_{2}(t, x)\right)^{-1 / 2} \frac{\partial g_{2}}{\partial x}(t, x) \text {. }
$$


A1.1 Assume first that $\partial g_{1} / \partial x=0$. Then by (55), it follows that $\partial g_{2} / \partial x=0$, which is possible only for $t=\sqrt{2} / 2$ and $x=\sqrt{2} / 4$. From (53), we have

$$
9 \operatorname{det} T_{3,1}(f) \geq G\left(\frac{\sqrt{2}}{2}, \frac{\sqrt{2}}{4}\right)=-\frac{17}{8}-\frac{3}{2} \sqrt{2}=-4.246320 \ldots
$$

A1.2 Assume now that $\partial g_{1} / \partial x \neq 0$. Then we can write the Eq. (55) as

$$
g_{2}(t, x)^{1 / 2}=\frac{\left(1-t^{2}\right) \frac{\partial g_{2}}{\partial x}(t, x)}{2 \frac{\partial g_{1}}{\partial x}(t, x)}=\frac{4\left(1-t^{2}\right)\left(-1+4 t^{2}-4 t x\right)}{-13+16 t^{2}+20 t x}
$$

or equivalently, by substituting (54), as

$$
\begin{aligned}
\Phi=\Phi(t, x):= & 3840 t^{8}+18944 t^{7} x+22528 t^{6} x^{2}+2560 t^{5} x^{3}-6400 t^{4} x^{4} \\
& +128 t^{6}-9472 t^{5} x-4992 t^{4} x^{2}+5120 t^{3} x^{3}-7552 t^{4} \\
& -2336 t^{3} x+1600 t^{2} x^{2}+3800 t^{2}-2000 t x+153=0 .
\end{aligned}
$$

Furthermore, note that by (56) the inequality

$$
0<\frac{\left(g_{2}(t, x)\right)^{1 / 2}}{4\left(1-t^{2}\right)}=\frac{-1+4 t^{2}-4 t x}{-13+16 t^{2}+20 t x}
$$

is true for

$$
0<t<\frac{\sqrt{2}}{2} \text { and } \frac{4 t^{2}-1}{4 t}<x<\frac{13-16 t^{2}}{20 t}
$$

or

$$
\frac{\sqrt{2}}{2}<t<1 \text { and }\left(x<\frac{4 t^{2}-1}{4 t} \text { or } x>-\frac{16 t^{2}-13}{20 t}\right) .
$$

Differentiating $G$ with respect to $t$ yields

$$
\frac{\partial G}{\partial t}(t, x)=\frac{\partial g_{1}}{\partial t}(t, x)+2 t\left(g_{2}(t, x)\right)^{1 / 2}-\frac{1}{2}\left(1-t^{2}\right)\left(g_{2}(t, x)\right)^{-1 / 2} \frac{\partial g_{2}}{\partial t}(t, x)
$$

By (56) and (59), we get

$$
\frac{\partial G}{\partial t}(t, x)=-\frac{36 t}{\left(-4 x t+4 t^{2}-1\right)\left(20 x t+16 t^{2}-13\right)} H(t, x),
$$

where

$$
H(t, x):=\left(-3+4 t^{2}+4 t x\right)\left(-11+6 t^{2}+8 t^{4}+2 t x+24 t^{3} x+40 t^{2} x^{2}\right) .
$$


Therefore, each critical point of $G$ satisfies

$$
-3+4 t^{2}+4 t x=0
$$

or

$$
-11+6 t^{2}+8 t^{4}+2 t x+24 t^{3} x+40 t^{2} x^{2}=0 .
$$

I. Assume that (60) holds. Then $x=x(t)=\left(3-4 t^{2}\right) /(4 t)$. Thus, by (57) we see that

$$
\Phi\left(t, \frac{3-4 t^{2}}{4 t}\right)=-8\left(48 t^{4}-104 t^{2}+39\right)\left(2 t^{2}-1\right)^{2}=0
$$

occurs only when $t=\hat{t}_{i}, i=1,2$, where

$$
\hat{t}_{1}:=\frac{1}{6} \sqrt{39-6 \sqrt{13}}=0.6945 \cdots, \quad \hat{t}_{2}:=\frac{\sqrt{2}}{2}=0.7071 \cdots
$$

Thus,

$$
x=x\left(\hat{t}_{1}\right)=\hat{x}_{1}=\frac{-2+\sqrt{13}}{\sqrt{39-6 \sqrt{13}}}=0.3852 \ldots
$$

and

$$
x=x\left(\hat{t}_{2}\right)=\hat{x}_{2}=\frac{\sqrt{2}}{4}=0.3535 \cdots
$$

However, it can be seen that

$$
\frac{-1+4 \hat{t}_{1}^{2}-4 \hat{t}_{1} \hat{x}_{1}}{-13+16 \hat{t}_{1}^{2}+20 \hat{t}_{1} \hat{x}_{1}}=-2<0
$$

which means that the inequality (58) is not satisfied for $t=\hat{t}_{1}$ and $x=\hat{x}_{1}$. Note that the case $t=\hat{t}_{2}$ and $x=\hat{x}_{2}$ reduces to A 1.1 .

Therefore, $G$ does not have critical point in the interior of $\Omega$.

II. Suppose now that (61) is satisfied. Equation (61) as a quadratic equation of $x$ with $\Delta:=441-216 t^{2}-176 t^{4}>0, t \in(0,1)$ has two roots, namely

$$
x_{i}=x_{i}(t)=\frac{-\left(1+12 t^{2}\right)+(-1)^{i+1} \sqrt{441-216 t^{2}-176 t^{4}}}{40 t}, \quad i=1,2 .
$$

a. Let $x=x_{1}$. Then $\Phi\left(t, x_{1}(t)\right)=0$ is equivalent to the equation

$$
\begin{gathered}
\left(-603-940 t^{2}-9936 t^{4}+7104 t^{6}\right) \sqrt{441-216 t^{2}-176 t^{4}} \\
=-2313-96696 t^{2}+20384 t^{4}-44928 t^{6}+92928 t^{8} .
\end{gathered}
$$


Squaring the both sides of (63) leads to

$$
518400 \gamma_{1}(t) \gamma_{2}^{2}(t)=0
$$

where for $t \in(0,1)$,

$$
\gamma_{1}(t):=-299+648 t^{2}+528 t^{4}
$$

and

$$
\gamma_{2}(t):=1+t^{2}-10 t^{4}+8 t^{6}=(1-t)(1+t)\left(1+4 t^{2}\right)\left(1-2 t^{2}\right) .
$$

Thus, there are two roots $t_{1}$ and $t_{2}$ in $(0,1)$ of the Eq. (64), namely

$$
t_{1}:=\frac{\sqrt{2}}{2}=0.707 \cdots, \quad t_{2}:=\frac{1}{66} \sqrt{-2673+2442 \sqrt{3}}=0.59779 \ldots .
$$

For $t=t_{1}$, by (62), $\tilde{x}_{1}:=x_{1}\left(t_{1}\right)=\sqrt{2} / 4$ and this case was discussed in A1.1.

For $t=t_{2}$, by (62),

$$
\tilde{x}_{2}:=x_{1}\left(t_{2}\right)=\frac{21+13 \sqrt{3}}{2 \sqrt{-2673+2442 \sqrt{3}}}=0.551477 \cdots
$$

It can be verified that $\tilde{\Phi}\left(t_{2}, \tilde{x}_{2}\right)=0$ and the inequality (58) holds for $t=t_{2}$ and $x=\tilde{x}_{2}$. Therefore, $G$ has a critical point at $\left(t_{2}, \tilde{x}_{2}\right)$.

b. Let $x=x_{2}$. Then $\Phi\left(t, x_{2}(t)\right)=0$ is equivalent to the equation

$$
\begin{aligned}
& -\left(-603-940 t^{2}-9936 t^{4}+7104 t^{6}\right) \sqrt{441-216 t^{2}-176 t^{4}} \\
& =-2313-96696 t^{2}+20384 t^{4}-44928 t^{6}+92928 t^{8} .
\end{aligned}
$$

Squaring both sides of (67) yields again the Eq. (64) having roots $t_{1}$ and $t_{2}$ given by (65), which do not satisfy (67).

Therefore, by A, B1 and B2, the function $G$ has a unique critical point at $\left(t_{2}, \tilde{x}_{2}\right)$. Denote

$$
\begin{aligned}
& \lambda_{1}:=\frac{\partial^{2} G}{\partial t^{2}}\left(t_{2}, \tilde{x}_{2}\right)=-\frac{30910188}{351923}+\frac{90657386}{1055769} \sqrt{3}=60.89649 \cdots, \\
& \lambda_{2}:=\frac{\partial^{2} G}{\partial t \partial x}\left(t_{2}, \tilde{x}_{2}\right)=-\frac{211055}{1177}+\frac{396373}{3531} \sqrt{3}, \\
& \lambda_{3}=\frac{\partial^{2} G}{\partial x^{2}}\left(t_{2}, \tilde{x}_{2}\right)=-\frac{308371}{1177}+\frac{551104}{3531} \sqrt{3} .
\end{aligned}
$$


Since $\lambda_{1}>0$ and

$$
\lambda_{1} \lambda_{3}-\lambda_{2}^{2}=-\frac{7933032}{1177}+\frac{4769748}{1177} \sqrt{3}=279.02623 \cdots>0,
$$

the function $G$ has a local minimum at $\left(t_{2}, \tilde{x}_{2}\right)$.

A2. It remains to consider $G$ in the boundary of $\Omega$.

(1) On the side $t=0$,

$$
G(0, x) \equiv 0>\Theta, \quad x \in[-1,1] .
$$

(2) On the side $t=1$,

$$
G(1, x)=-2+3 x+10 x^{2} \geq G\left(1,-\frac{3}{20}\right)=-\frac{89}{40}>\Theta, \quad x \in[-1,1] .
$$

(3) On the side $x=-1$,

$$
G(t,-1)=t\left(9+8 t-12 t^{2}\right) \geq G(0,-1)=0>\Theta, \quad t \in[0,1] .
$$

(4) On the side $x=1$,

$$
G(t, 1)=1-13 t+3 t^{2}+16 t^{3}+4 t^{4}-\left(1-t^{2}\right)\left|-1+4 t+4 t^{2}\right|=: \varrho(t) .
$$

When $t \in[0,(-1+\sqrt{2}) / 2]=: I_{1}$, we have $-1+4 t+4 t^{2} \leq 0$. Therefore, $\varrho(t)=t\left(-9+8 t+12 t^{2}\right) \geq G\left(\frac{\sqrt{2}-1}{2}, 1\right)=-\sqrt{2} \approx-1.41421>\Theta, \quad t \in I_{1}$.

When $t \in[(-1+\sqrt{2}) / 2,1]=: I_{2}$, we have $-1+4 t+4 t^{2} \geq 0$ and $\varrho(t)=$ $\left(-2+t+2 t^{2}\right)\left(-1+8 t+4 t^{2}\right)$. Since $\varrho^{\prime}(t)=0$ occurs only when $t=1 / 2 \in I_{2}$ and $\varrho^{\prime \prime}(1 / 2)=80>0$,we have

$$
\varrho(t) \geq \varrho\left(\frac{1}{2}\right)=-4>\Theta, \quad t \in I_{2}
$$

B. Suppose that $\zeta_{1}=0$. Then

$$
\Psi_{1}=10-8\left|\zeta_{2}\right|^{2}, \quad \Psi_{2}=-2 \operatorname{Re}\left(\zeta_{2}\right),
$$

and therefore,

$$
\operatorname{det} T_{3,1}(f)=\frac{1}{18}\left[10-8\left|\zeta_{2}\right|^{2}-2 \operatorname{Re}\left(\zeta_{2}\right)\right] \geq 0 .
$$


C. Suppose that $\zeta_{2}=0$ and $\zeta_{1} \neq 0$. Then

$$
\Psi_{1}=10-20\left|\zeta_{1}\right|^{2}+16\left|\zeta_{1}\right|^{4}, \quad \Psi_{2}=-26 \operatorname{Re} \zeta_{1}+10 \operatorname{Re}\left(\zeta_{1}^{2}\right)+32\left|\zeta_{1}\right|^{2} \operatorname{Re} \zeta_{1}
$$

Thus, taking $\zeta_{1}=r \mathrm{e}^{\mathrm{i} \theta}$, where $r \in(0,1]$ and $\theta \in[0,2 \pi)$ we have

$$
\Psi_{1}=10-20 r^{2}+16 r^{4} \quad \Psi_{2}=-26 r \cos \theta+10 r^{2} \cos 2 \theta+32 r^{3} \cos \theta .
$$

Then

$$
9 \operatorname{det} T_{3,1}(f)=G(r, \cos \theta), \quad r \in(0,1], \theta \in[0,2 \pi),
$$

where

$$
G(t, x):=5-15 t^{2}+8 t^{4}-13 t x+16 t^{3} x+10 t^{2} x^{2}
$$

for $t \in(0,1]$ and $x \in[-1,1]$. Set

$$
x_{w}=\frac{13-16 t^{2}}{20 t}, \quad t \in(0,1]
$$

Note that $-1<x_{w}$ occurs for $t \in(0,1)$ and $x_{w}<1$ holds for $t>(\sqrt{77}-5) / 8=$ $0.471870 \cdots$. Hence, for $t \in((\sqrt{77}-5) / 8,1)$ we have

$$
G(t, x) \geq G\left(t, x_{w}\right)=\frac{31}{40}-\frac{23}{5} t^{2}+\frac{8}{5} t^{4}=: \phi_{1}(t)
$$

Since $\phi_{1}$ is decreasing in $((\sqrt{77}-5) / 8,1)$,

$$
\phi_{1}(t) \geq-\frac{89}{40}=-2.225, \quad t \in\left(\frac{1}{8}(\sqrt{77}-5), 1\right) .
$$

On the other hand, $x_{w}>1$ occurs for $t \in(0,(\sqrt{77}-5) / 8)$. Hence,

$$
G(t, x) \geq G(t, 1)=5-13 t-5 t^{2}+16 t^{3}+8 t^{4}=: \phi_{2}(t) .
$$

Since $\phi_{2}$ is decreasing in $(0,(\sqrt{77}-5) / 8)$,

$$
\phi_{2}(t) \geq \frac{33}{64}-\frac{5}{64} \sqrt{77}=-0.169919 \ldots, \quad t \in\left(0, \frac{1}{8}(\sqrt{77}-5)\right) .
$$

Summarizing, form Parts A-C it follows that the inequality (44) holds.

It remains to show that the inequality (44) is sharp. It is observed from (45), (51), (52) and (53) that $9 \operatorname{det} T_{3,1}(f)=\Theta$ holds when the following conditions are satisfied:

$$
r=t_{2}, \quad \cos \theta=\tilde{x}_{2}, \quad s=1, \quad \sin (\psi+\alpha)=-1,
$$


where $t_{2}$ and $\tilde{x}_{2}$ are given by (65) and (66), and where $\alpha$ is determined by the condition (21) with $\kappa_{1}$ and $\kappa_{2}$ given by (50).

Now set $\theta=\operatorname{Arccos}\left(\tilde{x}_{2}\right)$ so that it satisfies the second condition in (68). Then $\kappa_{1}=3.309903 \cdots>0$ and $\kappa_{2}=-0.241293 \cdots<0$. Thus, (21) is satisfied if we take

$$
\alpha=-\operatorname{Arccos}\left(\frac{\kappa_{1}}{\sqrt{\kappa_{1}^{2}+\kappa_{2}^{2}}}\right)=-0.0727716 \cdots
$$

Thus, if we put

$$
\psi=\frac{3 \pi}{2}-\alpha=4.7851606 \cdots
$$

then $\psi$ satisfies the fourth condition in (68). Now consider a function $\tilde{p}$ which has the form (9) with $\zeta_{1}=t_{2} \mathrm{e}^{\mathrm{i} \theta}$ and $\zeta_{2}=\mathrm{e}^{\mathrm{i} \psi}$. Since $\zeta_{1} \in \mathbb{D}$ and $\zeta_{2} \in \mathbb{T}$, from Lemma 1 it follows that $\tilde{p} \in \mathcal{P}$, and so the extremal function $f$ in the class $\mathcal{F}_{2}$ for which equality in (44) holds satisfies (40) with $p:=\tilde{p}$.

Acknowledgements The authors thank the reviewers for theirs constructive comments and suggestions that helped to improve the clarity of this manuscript.

Funding None.

\section{Declarations}

Conflict of interest The authors declare that they have no conflict of interests.

Open Access This article is licensed under a Creative Commons Attribution 4.0 International License, which permits use, sharing, adaptation, distribution and reproduction in any medium or format, as long as you give appropriate credit to the original author(s) and the source, provide a link to the Creative Commons licence, and indicate if changes were made. The images or other third party material in this article are included in the article's Creative Commons licence, unless indicated otherwise in a credit line to the material. If material is not included in the article's Creative Commons licence and your intended use is not permitted by statutory regulation or exceeds the permitted use, you will need to obtain permission directly from the copyright holder. To view a copy of this licence, visit http://creativecommons.org/licenses/by/4.0/.

\section{References}

1. Ali, Md Firoz, Thomas, D.K., Vasudevarao, A.: Toeplitz determinants whose elements are the coefficients of analytic and univalent functions. Bull. Austr. Math. Soc. 97(2), 253-264 (2018)

2. Ali, M.F., Vasudevarao, A.: On logarithmic coefficients of some close-to-convex functions. Proc. Am. Math. Soc. 146, 1131-1142 (2018)

3. Biernacki, M.: Sur la représentation conforme des domaines linéairement accessibles. Prace Mat.-Fiz. 44, 293-314 (1936)

4. Carathéodory, C.: Über den Variabilitatsbereich der Koeffizienten von Potenzreihen, die gegebene werte nicht annehmen. Math. Ann. 64, 95-115 (1907) 
5. Cho, N.E., Kowalczyk, B., Kwon, O.S., Lecko, A., Sim, Y.J.: Some coefficient inequalities related to the Hankel determinant for strongly starlike functions of order alpha. J. Math. Inequal. 11(2), 429-439 (2017)

6. Cho, N.E., Kowalczyk, B., Kwon, O.S., Lecko, A., Sim, Y.J.: The bounds of some determinants for starlike functions of order alpha. Bull. Malays. Math. Sci. Soc. 41(1), 523-535 (2018)

7. Cho, N.E., Kowalczyk, B., Kwon, O.S., Lecko, A., Sim, Y.J.: On the third logarithmic coefficient in some subclasses of close-to-convex functions. Rev. R. Acad. Cienc. Exactas Fís. Nat. 114(52), 1-14 (2020)

8. Cho, N.E., Kowalczyk, B., Lecko, A.: Sharp bounds of some coefficient functionals over the class of functions convex in the direction of the imaginary axis. Bull. Aust. Math. Soc. 100, 86-96 (2019)

9. Cudna, K., Kwon, O.S., Lecko, A., Sim, Y.J., Śmiarowska, B.: The second and third-order Hermitian Toeplitz determinants for starlike and convex functions of order $\alpha$. Bol. Soc. Mat. Mex. 26, 361-375 (2020)

10. Goodman, A.W.: Univalent Functions. Mariner, Tampa (1983)

11. Jastrzȩbski, P., Kowalczyk, B., Kwon, O.S., Lecko, A., Sim, Y.J.: Hermitian Toeplitz determinants of the second and third-order for classes of close-to-star functions. Rev. R. Acad. Cienc. Exactas Fís. Nat. 114(166), 1-14 (2020)

12. Kaplan, W.: Close to convex schlicht functions. Mich. Math. J. 1, 169-185 (1952)

13. Kowalczyk, B., Kwon, O.S., Lecko, A., Sim, Y.J.: The bounds of some determinants for functions of bounded turning of order alpha. Bulletin de la Société Des Sciences et des Lettres de Łódź: Recherches sur les déformations LXVII(1), 107-118 (2017)

14. Kowalczyk, B., Kwon, O.S., Lecko, A., Sim, Y.J., Smiarowska, B.: The Third-Order Hermitian Toeplitz Determinant for Classes of Functions Convex in One Direction. Bull. Malays. Math. Sci. Soc. 43, 3143-3158 (2020)

15. Kowalczyk, B., Lecko, A., Sim, Y.J.: The sharp bound of the Hankel determinant of the third kind for convex functions. Bull. Aust. Math. Soc. 97, 435-445 (2018)

16. Kowalczyk, B., Lecko, A., Lecko, M., Sim, Y.J.: The sharp bound of the third Hankel determinant for some classes of analytic functions. Bull. Korean Math. Soc. 55(6), 1859-1868 (2018)

17. Kumar, U.P., Vasudevarao, A.: Logarithmic coefficients for certain subclasses of close-to-convex functions. Monatsh. Math. 187, 543-563 (2018)

18. Kwon, O.S., Lecko, A., Sim, Y.J.: The bound of the Hankel determinant of the third kind for starlike functions. Bull. Malays. Math. Sci. Soc. 42, 767-780 (2019)

19. Lecko, A., Sim, Y.J., Śmiarowska, B.: The sharp bound of the Hankel determinant of the third kind for starlike functions of order 1/2. Complex Anal. Oper. Theory 13, 2231-2238 (2019)

20. Lee, S.K., Ravichandran, V., Supramanian, S.: Bound for the second Hankel determinant of certain univalent functions. J. Inequal. Appl. 2013(281), 1-17 (2013)

21. Lecko, A.: A generalization of analytic condition for convexity in one direction. Demonstr. Math. XXX(1), 155-170 (2002)

22. Lewandowski, Z.: Sur l'identiteé de certaines classes de fonctions univalentes, I. Ann. Univ. Mariae Curie-Skłodowska Sect. A 12, 131-146 (1958)

23. Lewandowski, Z.: Sur l'identiteé de certaines classes de fonctions univalentes, II. Ann. Univ. Mariae Curie-Skłodowska Sect. A 14, 19-46 (1960)

24. Pommerenke, C.: Univalent Functions. Vandenhoeck \& Ruprecht, Göttingen (1975)

25. Ravichandran, V., Verma, S.: Generalized Zalcman conjecture for some classes of analytic functions. J. Math. Anal. Appl. 450(1), 592-605 (2017)

26. Robertson, M.S.: Analytic functions star-like in one direction. Am. J. Math. 58, 465-472 (1936)

Publisher's Note Springer Nature remains neutral with regard to jurisdictional claims in published maps and institutional affiliations. 